\title{
INTRODUCTION TO MODIFIED GRAVITY AND GRAVITATIONAL ALTERNATIVE FOR DARK ENERGY
}

\author{
Shin'ichi Nojiri* \\ Department of Applied Physics, National Defence Academy, Hashirimizu Yokosuka 239-8686, Japan \\ Sergei D. Odintsov ${ }^{\dagger}$ \\ Instituciò Catalana de Recerca i Estudis Avançats (ICREA) and Institut de Ciencies de l'Espai (IEEC-CSIC), \\ Campus UAB, Facultad Ciencies, Torre C5-Par-2a pl, E-08193 Bellaterra (Barcelona), Spain
}

\begin{abstract}
We review various modified gravities considered as gravitational alternative for dark energy. Specifically, we consider the versions of $f(R), f(G)$ or $f(R, G)$ gravity, model with non-linear gravitational coupling or string-inspired model with Gauss-Bonnet-dilaton coupling in the late universe where they lead to cosmic speed-up. It is shown that some of such theories may pass the Solar System tests. On the same time, it is demonstrated that they have quite rich cosmological structure: they may naturally describe the effective (cosmological constant, quintessence or phantom) late-time era with a possible transition from decceleration to acceleration thanks to gravitational terms which increase with scalar curvature decrease. The possibility to explain the coincidence problem as the manifestation of the universe expansion in such models is mentioned. The late (phantom or quintessence) universe filled with dark fluid with inhomogeneous equation of state (where inhomogeneous terms are originated from the modified gravity) is also described.
\end{abstract}

\section{INTRODUCTION}

The dark energy problem (for recent review see 1, 2] ) or, why current universe is expanding with the acceleration, is considered to be the one of the most fundamental theoretical problems of XXI century. There are various directions aimed to construct the acceptable dark energy model. Specifically, one can mention scalar (quintessence or phantom) models, dark fluid with complicated equation of state (EoS), more complicated field theories with fermions, abelian or non-abelian vector field, string/M-theory, higher dimensions, etc. Nevertheless, despite the number of attempts still there is no any satisfactory explanation of dark energy origin. This is understandable having in mind that even current values of cosmological parameters are not yet defined with precise accuracy, and even less is known about their evolution.

The modified gravity approach is extremely attractive in the applications for late accelerating universe and dark energy. Indeed,

1. Modified gravity provides the very natural gravitational alternative for dark energy. The cosmic speed-up is explained simply by the fact of the universe expansion where some sub-dominant terms (like $1 / R$ ) may become essential at small curvature.

2. Modified gravity presents very natural unification of the early-time inflation and late-time acceleration thanks to different role of gravitational terms relevant at small and at large curvature. Moreover, some models of modified gravity are predicted by string/M-theory considerations.

3. It may serve as the basis for unified explanation of dark energy and dark matter. Some cosmological effects (like galaxies rotation curves) may be explained in frames of modified gravity.

4. Assuming that universe is entering the phantom phase, modified gravity may naturally describe the transition from non-phantom phase to phantom one without necessity to introduce the exotic matter (like the scalar with wrong sign kinetic term or ideal fluid with EoS parameter less than -1). In addition, often the phantom phase in modified gravity is transient. Hence, no future Big Rip is usually expected there.

5. Modified gravity quite naturally describes the transition from decceleration to acceleration in the universe evolution. 6. The effective dark energy dominance may be assisted by the modification of gravity. Hence, the coincidence problem is solved there simply by the fact of the universe expansion.

7. Modified gravity is expected to be useful in high energy physics (for instance, for the explanation of hierarchy problem or unification of GUTs with gravity).

8. Despite quite stringent constraints from Solar System tests, there are versions of modified gravity which may be viable theories competing with General Relativity at current epoch. Nevertheless, more serious check of such theories

\footnotetext{
* Address from April: Dept. of Phys., Nagoya Univ., Nagoya 464-8602, Japan. E-mail: snojiri@yukawa.kyoto-u.ac.jp, nojiri@cc.nda.ac.jp

$\dagger$ also at TSPU, Tomsk, E-mail: odintsov@ieec.uab.es
} 
is necessary to fit them with various observational data and precise Solar System tests.

These lectures are devoted to the review of some modified gravities with above mentioned attractive features and their cosmological properties at late universe. We do not discuss here modified gravities related with brane-world approach as well as Palatini formulation of modified gravities.

The paper is organized as follows. In sect $\amalg$ we start from general $f(R)$ gravity in accelerating FRW universe. It is shown how this theory may be rewritten as scalar-tensor gravity (Einstein frame). Two specific models: the one with $1 / R$ and $R^{m}$ terms, and another with $\ln R$ terms are considered. It is shown that both models may lead to the (cosmological constant or quintessence) acceleration of the universe as well as an early time inflation. Moreover, the first model seems to pass the Solar System tests, i.e. it has the acceptable newtonian limit, no instabilities and no Brans-Dicke problem (decoupling of scalar) in scalar-tensor version. The coupling of such modified gravity with usual matter is considered. It is shown the occurence of transient phantom era in this case. General conditions to $f(R)$ gravity admitting phantom divide crossing are derived. Finally, it is demonstrated how scalar-tensor theory may be rewritten as some version of modified gravity. Sect $\amalg$ in devoted to the study of FRW cosmology in string-inspired dilaton gravity containing scalar-Gauss-Bonnet coupling. The occurence of acceleration is again mentioned. In sect IV the non-linear coupling of gravity with matter is considered. It is shown that some forms of such coupling may help to solve the coincidence problem simply by the fact of the universe expansion. From another point, such modified gravity may also predict (cosmological constant, quintessence or phantom) accelerating epoch. It is interesting that the model with such non-linear coupling may serve for quite interesting dynamical solution of cosmological constant problem. The possible applications of modified gravity for solution of hierarchy problem of high energy physics are described.

In sect $\square$ we consider $f(G)$ and $f(R, G)$ theories where $G$ is Gauss-Bonnet invariant. It is shown the possibility of cosmic speed-up for various choices of function $f$, as well as possibility of decceleration-acceleration transition. Sect $[\mathrm{VI}$ is devoted to the study of late-time cosmology for the universe filled with dark fluid with inhomogeneous EoS. At first, the structure of future singularities is classified for dark fluid with homogeneous EoS: $p=-\rho+A \rho^{\alpha}$. Next, the impact of the inhomogeneous terms to future singularities structure is analyzed for the same (effectively, phantom) dark fluid. As the motivation for inhomogeneous EoS dark fluid, the possible origin of the inhomogeneous terms from scalar-tensor or modified gravity is indicated. Some outlook and discussion are given in the last section.

\section{THE MODIFIED $f(R)$ GRAVITY}

In the present section $f(R)$ gravity in FRW universe is considered. Special attention is paid to the versions of such theory which may describe cosmic speed-up at late universe. Let us start from the rather general 4-dimensional action:

$$
S=\int d^{4} x \sqrt{-g}\left\{f(R)+L_{m}\right\}
$$

Here $R$ is the scalar curvature, $f(R)$ is an arbitrary function and $L_{m}$ is a matter Lagrangian density. The equation of the motion is given by

$$
0=\frac{1}{2} g_{\mu \nu} f(R)-R_{\mu \nu} f^{\prime}(R)-\nabla_{\mu} \nabla_{\nu} f^{\prime}(R)-g_{\mu \nu} \nabla^{2} f^{\prime}(R)+\frac{1}{2} T_{\mu \nu} .
$$

With no matter and for the Ricci tensor $R_{\mu \nu}$ being covariantly constant, the equation of motion corresponding to the action (11) is:

$$
0=2 f(R)-R f^{\prime}(R)
$$

which is the algebraic equation with respect to $R$. If the solution of Eq.(3) is positive, it expresses deSitter universe and if negative, anti-deSitter universe. In the following, the metric is assumed to be in the FRW form:

$$
d s^{2}=-d t^{2}+\hat{a}(t)^{2} \sum_{i=1}^{3}\left(d x^{i}\right)^{2} .
$$

Here we assume that the spatial part is flat as suggested by the observation of the Cosmic Microwave Background (CMB) radiation. Without the matter and in FRW background, Eq.(2) gives

$$
0=-\frac{1}{2} f(R)+3\left(H^{2}+\dot{H}\right) f^{\prime}(R)-6 \frac{\dot{H}}{H} f^{\prime \prime}(R)-18 H^{2} \frac{d}{d t}\left(\frac{\dot{H}}{H^{2}}\right) f^{\prime \prime}(R) .
$$


Here $R$ is given by $R=12 H^{2}+6 \dot{H}$. Our main purpose is to look for accelerating cosmological solutions of the following form: de Sitter $(\mathrm{dS})$ space, where $H$ is constant and $a(t) \propto \mathrm{e}^{H t}$, quintessence and phantom like cosmologies:

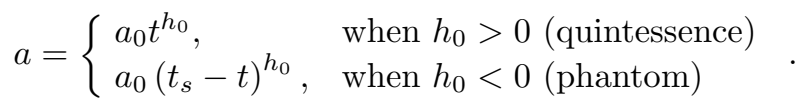

The cosmologies of above type in various modified gravity models will be often discussed in this work.

Introducing the auxiliary fields, $A$ and $B$, one can rewrite the action (1) as follows:

$$
S=\int d^{4} x \sqrt{-g}\left[\frac{1}{\kappa^{2}}\{B(R-A)+f(A)\}+\mathcal{L}_{\text {matter }}\right] .
$$

One is able to eliminate $B$, and to obtain

$$
S=\int d^{4} x \sqrt{-g}\left[\frac{1}{\kappa^{2}}\left\{f^{\prime}(A)(R-A)+f(A)\right\}+\mathcal{L}_{\text {matter }}\right],
$$

and by using the conformal transformation $g_{\mu \nu} \rightarrow \mathrm{e}^{\sigma} g_{\mu \nu}\left(\sigma=-\ln f^{\prime}(A)\right)$, the action (8) is rewritten as the Einsteinframe action:

$$
S_{E}=\int d^{4} x \sqrt{-g}\left[\frac{1}{\kappa^{2}}\left(R-\frac{3}{2} g^{\rho \sigma} \partial_{\rho} \sigma \partial_{\sigma} \sigma-V(\sigma)\right)+\mathcal{L}_{\text {matter }}^{\sigma}\right]
$$

Here,

$$
V(\sigma)=\mathrm{e}^{\sigma} G\left(\mathrm{e}^{-\sigma}\right)-\mathrm{e}^{2 \sigma} f\left(G\left(\mathrm{e}^{-\sigma}\right)\right)=\frac{A}{f^{\prime}(A)}-\frac{f(A)}{f^{\prime}(A)^{2}} .
$$

The action (8) is called the Jordan-frame action. In the Einstein-frame action, the matter couples with the scalar field $\sigma$. Due to the scale transformation, there is a difference in time interval between the Einstein and Jordan frames. Of course, we usually use clock to measure time. The clock could be based essentially on electromagnetism. In the Jordan frame, where the scalar field $\sigma$ does not couple with matter, especially with electromagnetic fields, the time measured by the clock is proportional to the cosmological time in the FRW metric. On the other hand, in the Einstein frame where the scalar field $\sigma$ couples with matter, the time measured by the clock is not always proportional to the cosmological time, due to the coupling of the scalar field $\sigma$ with electromagnetic field. The difference, of course, comes from the factor of the scale transformation, which includes the scalar field $\sigma$. Since $\sigma$ is time-dependent in general, the time measured by the clock becomes, say, slower than the cosmological time in the Einstein frame. It is estimated the acceleration of the universe started about five billion years ago. The five billion years are measured by using the electromagnetism. Hence, these five billion years could be identified with five billion years in the Jordan frame but not with those in the Einstein frame (for further discussion of the relation between Einstein and Jordan frames in cosmology, see review [3]).

One may define the effective EoS parameter $w_{\text {eff }}$ in Jordan frame as

$$
w_{\mathrm{eff}}=\frac{p}{\rho}=-1-\frac{2 \dot{H}}{3 H^{2}}
$$

The scale factor in Einstein frame (when the two frames appear) is denoted as $a(t)$. Note that if we dont make transformation from one frame to another one, then scale factor will be always denoted without hat.

\section{A. Modified gravity with negative and positive powers of the curvature}

As the first gravitational alternative for dark energy we consider the following action [4]

$$
f(R)=R-\frac{c}{\left(R-\Lambda_{1}\right)^{n}}+b\left(R-\Lambda_{2}\right)^{m} .
$$

Here we assume the coefficients $n, m, c, b>0$ but $n, m$ may be fractional. Let us show that above model leads to acceptable cosmic speed-up and is consistent with Solar System tests. 
For the action (12), Eq.(3) has the following form:

$$
0=-R+\frac{(n+2) c}{\left(R-\Lambda_{1}\right)^{n}}+(m-2) b\left(R-\Lambda_{2}\right)^{m} .
$$

Especially when $n=1$ and $m=2$, one gets

$$
R=R_{ \pm}=\frac{\Lambda_{1} \pm \sqrt{\Lambda_{1}^{2}+12 c}}{2}
$$

If $c>0$, one solution corresponds to deSitter space and another to anti-deSitter. If $-\frac{\Lambda_{1}^{2}}{12}<c<0$ and $\Lambda_{1}>0$, both of solutions express the deSitter space. Hence, the natural possibility for the unification of early-time inflation with late-time acceleration appears (compare with proposal [5]). Here, higher derivative terms act in favour of early-time inflation while $1 / R$ term supports cosmic speed-up.

By assuming the FRW universe metric (44), one may define the Hubble rate by $H=\dot{\hat{a}} / \hat{a}$. The contribution from matter may be neglected. Especially when $n=1, m=2$, and $\Lambda_{1}=\Lambda_{2}=0$ in (12) and the curvature is small, we obtain the following solution of (2]): $\hat{a} \propto t^{2}$, which is consistent with the result in [6]. If the present universe expands with above power law, the curvature of the present universe should be small compared with that of the deSitter universe solution in (14) with $\Lambda_{1}=0$. As the Hubble rate in the present universe is $\left(10^{-33} \mathrm{eV}\right)^{-1}$, the parameter $c$, which corresponds to $\mu^{4}$ in $\underline{\underline{6}}$, should be much larger than $\left(10^{-33} \mathrm{eV}\right)^{4}$.

We now consider the more general case that $f(R)$ is given by (12) when the curvature is small. Neglecting the contribution from the matter again, solving (2), we obtain $\hat{a} \propto t^{\frac{(n+1)(2 n+1)}{n+2}}$. It is quite remarkable that actually any negative power of the curvature supports the cosmic acceleration. This gives the freedom in modification of the model to achieve the consistency with experimental tests of newtonian gravity.

On the other hand, when the scalar curvature $R$ is large, one obtains $\hat{a} \propto t^{-\frac{(m-1)(2 m-1)}{m-2}}$. When $\frac{(m-1)(2 m-1)}{m-2}>0$, the universe is shrinking but if we change the arrow of time by $t \rightarrow t_{s}-t$, the inflation occurs with the inverse power law and at $t=t_{s}$, the size of the universe diverges. It is remarkable that when $m$ is fractional (or irrational) and $1<m<2$, the expression of $\hat{a}$ is still valid and the power becomes positive, the universe evolves with the (fractional) power law expansion.

It is interesting that the model of this section seems to be more consistent than the one of ref. [6] (for discussion of newtonian limit in $1 / R$ theory see $7 \mid$ ) as it may pass Solar System tests. Indeed, in [8] , small gravitational object like the Earth or the Sun in the model $[\underline{6}]$ is considered. It has been shown that the system becomes instable. This may cause the unacceptable force between distant Galaxies [9]. As shown in [4], however, by adding the positive power (higher than 1) of the scalar curvature to the action, the instability could be significally improved. Moreover, the account of quantum effects in modified gravity also acts against instability [10].

It has been also mentioned in [1] that $1 / R$ model [6] which is equivalent to some scalar-tensor gravity is ruled out as realistic theory due to the constraints to such Brans-Dicke type theories. In [4], it was shown that by adding the scalar curvature squared term to the action, the mass of the scalar field can be adjusted to be very large and the scalar field can decouple. Hence, the modified gravity theory of this section (after some fine-tuning [4]) passes the Solar System tests. In addition, at precisely the same values of parameters the above modified gravity has the newtonian limit which does not deviate significally from the one in General Relativity.

\section{B. $\quad \ln R$ gravity}

Other gravitational alternatives for dark energy may be suggested along the same line. As an extension of the theory of the previous section, one may consider the model [12] containing the logarithm of the scalar curvature $R$ :

$$
f(R)=R+\alpha^{\prime} \ln \frac{R}{\mu^{2}}+\beta R^{m} .
$$

We should note that $m=2$ choice simplifies the model. Assuming the scalar curvature is constant and the Ricci tensor is also covariantly constant, the equations (3) are:

$$
0=2 f(R)-R f^{\prime}(R)=\tilde{f}(R) \equiv R+2 \alpha^{\prime} \ln \frac{R}{\mu^{2}}-\alpha^{\prime} .
$$

If $\alpha^{\prime}>0, \tilde{f}(R)$ is monotonically increasing function and $\lim _{R \rightarrow 0} \tilde{f}(R)=-\infty$ and $\lim _{R \rightarrow+\infty} \tilde{f}(R)=+\infty$. There is one and only one solution of (16). This solution may correspond to the inflation. On the other hand, if $\alpha^{\prime}<0$, 
$\lim _{R \rightarrow 0} \tilde{f}(R)=\infty$ and $\lim _{R \rightarrow+\infty}=+\infty$. Since $\tilde{f}^{\prime}(R)=1+\frac{\alpha^{\prime}}{R}$, the minimum of $\tilde{f}(R)$, where $\tilde{f}^{\prime}(R)=0$, is given by $R=-2 \alpha^{\prime}$. If $\tilde{f}\left(-2 \alpha^{\prime}\right)>0$, there is no solution of (16). If $\tilde{f}\left(-2 \alpha^{\prime}\right)=0$, there is only one solution and if $\tilde{f}\left(-2 \alpha^{\prime}\right)<0$, there are two solutions. Since $\tilde{f}\left(-2 \alpha^{\prime}\right)=-2 \alpha^{\prime}\left(1-\ln \frac{-2 \alpha^{\prime}}{\mu^{2}}\right)$, there are two solutions if $-\frac{2 \alpha^{\prime}}{\mu^{2}}>$ e. Since the square root of the curvature $R$ corresponds to the rate of the expansion of the universe, the larger solution in two solutions might correspond to the inflation in the early universe and the smaller one to the present accelerating universe.

We can consider late FRW cosmology when the scalar curvature $R$ is small. Solving (2), it follows that the power law inflation could occur: $\hat{a} \propto t^{\frac{1}{2}}$. Since $\dot{\hat{a}}>0$ but $\ddot{\hat{a}}<0$, the deccelerated expansion occurs.

One may discuss further generalizations like [12] (see also [13])

$$
f(R)=R+\gamma R^{-n}\left(\ln \frac{R}{\mu^{2}}\right)^{m} .
$$

Here $n$ is rectricted by $n>-1$ ( $m$ is an arbitrary) in order that the second term could be more dominant than the Einstein term when $R$ is small. For this model, we find

$$
\hat{a} \sim t^{\frac{(n+1)(2 n+1)}{n+2}} .
$$

This does not depend on $m$. The logarithmic factor is almost irrelevant. The effective $w_{\text {eff }}$ is given by

$$
w_{\text {eff }}=-\frac{6 n^{2}+7 n-1}{3(n+1)(2 n+1)} .
$$

Then $w_{\text {eff }}$ can be negative if

$$
-1<n<-\frac{1}{2} \text { or } n>\frac{-7+\sqrt{73}}{12}=0.1287 \cdots .
$$

From (18), the condition that the universe could accelerate is $\frac{(n+1)(2 n+1)}{n+2}>1$, that is:

$$
n>\frac{-1+\sqrt{3}}{2}=0.366 \cdots
$$

Clearly, the effective EoS parameter $w$ may be within the existing bounds.

\section{Modified gravity coupled with matter}

In this subsection we will show that the modified gravity may lead to the effective phantom or quintessence dark energy without necessity to introduce the (negative kinetic energy) phantom scalar or negative pressure ideal fluid. In fact, the matter is taken to be the usual ideal fluid.

The ideal fluid is taken as the matter with the constant $w: p=w \rho$. Then from the energy conservation law it follows $\rho=\rho_{0} a^{-3(1+w)}$. In a some limit, strong cuvature or weak one, $f(R)$ may behave as $f(R) \sim f_{0} R^{\alpha}$, with constant $f_{0}$ and $\alpha$. An exact solution of the equation of motion is found to be [14]

$$
\begin{aligned}
& a=a_{0} t^{h_{0}}, \quad h_{0} \equiv \frac{2 \alpha}{3(1+w)} \\
& a_{0} \equiv\left[-\frac{6 f_{0} h_{0}}{\rho_{0}}\left(-6 h_{0}+12 h_{0}^{2}\right)^{\alpha-1}\left\{(1-2 \alpha)(1-\alpha)-(2-\alpha) h_{0}\right\}\right]^{-\frac{1}{3(1+w)}} .
\end{aligned}
$$

When $\alpha=1$, the result $h_{0}=\frac{2}{3(1+w)}$ in the Einstein gravity is reproduced. The effective $w_{\text {eff }}$ may be defined by $h_{0}=\frac{2}{3\left(1+w_{\text {eff }}\right)}$. By using (22), one finds the effective $w_{\text {eff }}$ (11) is given by

$$
w_{\mathrm{eff}}=-1+\frac{1+w}{\alpha}
$$

Hence, if $w$ is greater than -1 (effective quintessence or even usual ideal fluid with positive $w$ ), when $\alpha$ is negative, we obtain the effective phantom phase where $w_{\text {eff }}$ is less than -1 . This is different from the case of pure modified gravity. Moreover, when $\alpha>w+1$ (it can be even positive), $w_{\text {eff }}$ could be negative (for negative $w$ ). Hence, it follows that 
modified gravity minimally coupled with usual (or quintessence) matter may reproduce quintessence (or phantom) evolution phase for dark energy universe in an easier way than without such coupling.

One may now take $f(R)$ as

$$
f(R)=\frac{1}{\kappa^{2}}\left(R-\gamma R^{-n}+\eta R^{2}\right)
$$

When the cuvature is small, the second term becomes dominant and one may identify $f_{0}=-\frac{\gamma}{\kappa^{2}}$ and $\alpha=-n$. Then from (23), it follows $w_{\text {eff }}=-1-\frac{1+w}{n}$. Hence, if $n>0$, an effective phantom era occurs even if $w>-1$. Usually the phantom era generates the future Big Rip singularity [15]. (Note that there may occur different types of future singularities, say, sudden ones [16, 17, 18, 19], etc. The complete classification of Big Rip singularities is given in [18.) However, near the Big Rip singularity, the curvature becomes large and the last term becomes dominant. In this case $\alpha=2$ and $w_{\text {eff }}=\frac{-1+w}{2}$. Then if $w>-1$, it follows $w_{\text {eff }}>-1$, which prevents the Big Rip singularity (it makes the phantom phase transient). To conclude, it looks quite promising that modest modification of General Relativity coupled to ideal fluid matter leads to an effective dark energy universe in the very natural way.

\section{The equivalence with scalar-tensor theory}

It is very interesting that $f(R)$ gravity is in some sense equivalent to the scalar-tensor theory with the action:

$$
\begin{gathered}
S=\int d^{4} x \sqrt{-g}\left\{\frac{1}{2 \kappa^{2}} R-\frac{1}{2} \omega(\phi) \partial_{\mu} \phi \partial^{\mu} \phi-V(\phi)\right\}, \\
\omega(\phi)=-\frac{2}{\kappa^{2}} h^{\prime}(\phi), \quad V(\phi)=\frac{1}{\kappa^{2}}\left(3 h(\phi)^{2}+h^{\prime}(\phi)\right) .
\end{gathered}
$$

Here $h(\phi)$ is a proper function of the scalar field $\phi$. Imagine the following FRW cosmology is constructed:

$$
\phi=t, \quad H=h(t) .
$$

Then, as it has been demonstrated in ref. 20], any cosmology defined by $H=h(t)$ in (26) can be realized by (25). Indeed, if one defines a new field $\varphi$ as

$$
\varphi=\int d \phi \sqrt{|\omega(\phi)|}
$$

the action (25) can be rewritten as

$$
S=\int d^{4} x \sqrt{-g}\left\{\frac{1}{2 \kappa^{2}} R \mp \frac{1}{2} \partial_{\mu} \varphi \partial^{\mu} \varphi-\tilde{V}(\varphi)\right\}
$$

The sign in front of the kinetic term depends on the sign of $\omega(\phi)$. If the sign of $\omega$ and therefore the sign of $\dot{H}$ is positive (negative), the sign of the kinetic term is $-(+)$. Therefore, in the phantom phase, the sign is always + and in the non-phantom phase, always -. One assumes $\phi$ can be solved with respect to $\varphi: \phi=\phi(\varphi)$. Then the potential $\tilde{V}(\varphi)$ is given by $\tilde{V}(\varphi) \equiv V(\phi(\varphi))$. Since $\tilde{V}(\varphi)$ could be uniquely determined, there is one-to-one correspondence between $H$ and $\tilde{V}(\varphi)$.

In case the sign in front of the kinetic term of $\varphi$ in (28) is - , we can use the conformal transformation $g_{\mu \nu} \rightarrow$ $\mathrm{e}^{ \pm \kappa \varphi \sqrt{\frac{2}{3}}} g_{\mu \nu}$, and make the kinetic term of $\varphi$ vanish. Hence, one obtains

$$
S=\int d^{4} x \sqrt{-g}\left\{\frac{\mathrm{e}^{ \pm \kappa \varphi \sqrt{\frac{2}{3}}}}{2 \kappa^{2}} R-\mathrm{e}^{ \pm 2 \kappa \varphi \sqrt{\frac{2}{3}}} \tilde{V}(\varphi)\right\}
$$

The action (29) may be called as Jordan frame action and the action (28) as the Einstein frame action. Since $\varphi$ becomes the auxiliary field, one may delete $\varphi$ by using an equation of motion:

$$
R=\mathrm{e}^{ \pm \kappa \varphi \sqrt{\frac{2}{3}}}\left(4 \kappa^{2} \tilde{V}(\varphi) \pm 2 \kappa \sqrt{\frac{3}{2}} \tilde{V}^{\prime}(\varphi)\right)
$$


which may be solved with respect to $R$ as $\varphi=\varphi(R)$. One can rewrite the action (29) in the form of $f(R)$ gravity [20]:

$$
\begin{aligned}
S & =\int d^{4} x \sqrt{-g} f(R), \\
f(R) & \equiv \frac{\mathrm{e}^{ \pm \kappa \varphi(R) \sqrt{\frac{2}{3}}}}{2 \kappa^{2}} R-\mathrm{e}^{ \pm 2 \kappa \varphi(R) \sqrt{\frac{2}{3}}} \tilde{V}(\varphi(R)) .
\end{aligned}
$$

Note that one can rewrite the scalar-tensor theory (25) or equivalently (28), only when the sign in front of the kinetic term is - in (28), that is, $\omega(\phi)$ is positive. In the phantom phase, $\omega(\phi)$ is negative. In this case, the above trick to rewrite the phantom scalar-tensor theory as $f(R)$ gravity does not work. We should note, however, as the metric is conformally transformed, even if the universe in the original metric (corresponding to the Einstein frame) is in the phantom phase, the universe in the rescaled metric (corresponding to the Jordan frame) can be in non-phantom phase in general. In addition, one should bear in mind that the same FRW dynamics (same scale factor) may be obtained from one of these two mathematically equivalent descriptions. Nevertheless, the properties of such FRW cosmology in both models may be quite different (for instance, more FRW solutions may exist, say, for scalar-tensor theory, newton law may be different, stability of such equivalent solutions may be different, etc).

\section{E. Transition from phantom to non-phantom phase}

Assuming that our universe is entering into the phantom phase recently, and having in mind the occurence of (cosmologically recent) decceleration-acceleration transition, the question appears: what are the conditions for phantomnon-phantom transition in modified $f(R)$ gravity? For instance, the conditions for the existence of deSitter universe solution in $f(R)$ gravity may be derived in the form of algebraic equation [21]. The stability of deSitter universe solution was established in ref.[21] where one-loop quantization of $f(R)$ was done. (The same stability criteria was also found in ref. 22] using perturbations analysis.)

The effective EoS parameter is defined by (11). We now define a time-dependent parameter $F(t)$ by

$$
F(t) \equiv-\frac{2 \dot{H}}{3 H^{2}}
$$

that is, $F(t)=1+w_{\text {eff }}$. Note that $F=0$ at the transition point between non-phantom $\left(w_{\text {eff }}>-1\right.$ or $\left.F>0\right)$ and phantom $\left(w_{\text {eff }}<-1\right.$ or $\left.F<0\right)$ phases.

We now consider the region near the transition point between non-phantom and phantom phases and assume $F$ is small. Then Eq.(5) reduces to

$$
0=-\frac{1}{2} f\left(12 H^{2}\right)+3 H^{2} f^{\prime}\left(12 H^{2}\right)+81 H^{4} F f^{\prime \prime}\left(12 H^{2}\right)+27 H^{3} \dot{F} f^{\prime \prime}\left(12 H^{2}\right)+\mathcal{O}\left(F^{2}\right) .
$$

If the transition point corresponds to the deSitter solution, which satisfies

$$
0=-\frac{1}{2} f\left(12 H^{2}\right)+3 H^{2} f^{\prime}\left(12 H^{2}\right),
$$

one finds $\dot{F} \sim-3 H F$. Then $F$ behaves as $F \sim F_{0} \mathrm{e}^{-3 H t}$, which does not vanish in a finite time and therefore there does not occur the transition. On the other hand, if the transition point does NOT correspond to the deSitter solution, it follows

$$
\dot{F} \sim \frac{f\left(12 H^{2}\right)-6 H^{2} f^{\prime}\left(12 H^{2}\right)}{54 H^{3} f^{\prime \prime}\left(12 H^{2}\right)}
$$

Then near the transition point if

$$
\begin{aligned}
f^{\prime \prime}\left(12 H^{2}\right) & >0 \text { and } f\left(12 H^{2}\right)-6 H^{2} f^{\prime}\left(12 H^{2}\right)<0 \\
\text { or } \quad f^{\prime \prime}\left(12 H^{2}\right) & <0 \text { and } f\left(12 H^{2}\right)-6 H^{2} f^{\prime}\left(12 H^{2}\right)>0,
\end{aligned}
$$

there could occur the transition from the non-phantom phase $(F>0)$ to phantom phase $(F<0)$.

As an example, we consider

$$
f(R)=f_{0} \mathrm{e}^{R / 6 H_{0}^{2}},
$$


with constants $f_{0}$ and $H_{0}$ (for similar choice with negative $R$ in the exponent see [23].) From (35]), one finds

$$
\dot{F} \sim \frac{2 H_{0}^{2}\left(H_{0}^{2}-H^{2}\right)}{3 H^{3}}
$$

Then by properly choosing initial conditions, if $H>H_{0}$ when $F \sim 0$ but positive (that is, in non-phantom phase), we find $\dot{F}<0$ and there could occur the transition from non-phantom phase to phantom phase (crossing of phantom divide).

\section{STRING-INSPIRED GAUSS-BONNET GRAVITY AS DARK ENERGY}

The successful dark energy theory may be searched in string/M-theory. Indeed, it is quite possible that some unusual gravity-matter couplings predicted by the fundamental theory may become important at current, low-curvature universe (being not essential in intermediate epoch from strong to low curvature). For instance, in the study of stringinduced gravity near to initial singularity the role of Gauss-Bonnet (GB) coupling with scalar was quite important for ocurrence of non-singular cosmology [24, 25]. Moreover, string/M-theory naturally predicts the appearence of the terms with inverse powers of curvature invariants in compactified, low-energy effective action [26].

The present section is devoted to the study of the role of GB coupling with the scalar field (which naturally appears in low-energy effective action) to the late-time universe 27]. It is explicitly demonstrated that such term itself cannot induce the effective phantom late-time universe if the scalar is canonical in the absence of potential term. It may produce the effective quintessence (or phantom) era, explaining the current acceleration only when the scalar is phantom or when the scalar is canonical with non-zero potential. It is interesting that it may also have the important impact to the Big Rip singularity, similarly to quantum effects [17, 18, 28], preventing it. Note that we concentrate mainly on the exponential scalar-GB coupling and exponential scalar potential, while the consideration of other types of such functions and their role in late time cosmology may be considered too [29].

We consider a model of the scalar field $\phi$ coupled with gravity. As a stringy correction, the term proportional to the GB invariant $G=R^{2}-4 R_{\mu \nu} R^{\mu \nu}+R_{\mu \nu \rho \sigma} R^{\mu \nu \rho \sigma}$ is added. The starting action is given by

$$
\begin{aligned}
& S=\int d^{4} x \sqrt{-g}\left\{\frac{1}{2 \kappa^{2}} R-\frac{\gamma}{2} \partial_{\mu} \phi \partial^{\mu} \phi-V(\phi)+f(\phi) G\right\}, \\
& V=V_{0} \mathrm{e}^{-\frac{2 \phi}{\phi_{0}}}, \quad f(\phi)=f_{0} \mathrm{e}^{\frac{2 \phi}{\phi_{0}}} .
\end{aligned}
$$

Here $\gamma= \pm 1$. For the canonical scalar, $\gamma=1$ but at least when GB term is not included, the scalar behaves as phantom only when $\gamma=-1$.

Starting with FRW universe metric (11) in sect II and assuming (6) in sect the following solutions may be obtained

$$
\begin{aligned}
V_{0} t_{1}^{2} & =-\frac{1}{\kappa^{2}\left(1+h_{0}\right)}\left\{3 h_{0}^{2}\left(1-h_{0}\right)+\frac{\gamma \phi_{0}^{2} \kappa^{2}\left(1-5 h_{0}\right)}{2}\right\} \\
\frac{48 f_{0} h_{0}^{2}}{t_{1}^{2}} & =-\frac{6}{\kappa^{2}\left(1+h_{0}\right)}\left(h_{0}-\frac{\gamma \phi_{0}^{2} \kappa^{2}}{2}\right)
\end{aligned}
$$

Even if $\gamma=-1$, there appear the solutions describing non-phantom cosmology coresponding the quintessence or matter. As an example, we consider the case that $h_{0}=-\frac{80}{3}<-1$, which gives $w_{\text {eff }}=-1.025$. Simple tuning gives other acceptable values of the effective $w$ in the range close to -1 . This is consistent with the observational bounds for effective $w$ (for recent discussion and complete list of refs., see [30]). Then from (40), one obtains

$$
V_{0} t_{1}^{2}=\frac{1}{\kappa^{2}}\left(\frac{531200}{231}+\frac{403}{154} \gamma \phi_{0} \kappa^{2}\right), \quad \frac{f_{0}}{t_{1}^{2}}=-\frac{1}{\kappa^{2}}\left(\frac{9}{49280}+\frac{27}{7884800} \gamma \phi_{0} \kappa^{2}\right) .
$$

Therefore even starting from the canonical scalar theory with positive potential, we may obtain a solution which reproduces the observed value of $w$.

There is another kind of solutions [27]. If $\phi$ and $H$ are constants: $\phi=\varphi_{0}, H=H_{0}$, this corresponds to deSitter space. Then the solution of equations of motion gives:

$$
H_{0}^{2}=-\frac{\mathrm{e}^{-\frac{2 \varphi_{0}}{\phi_{0}}}}{8 f_{0} \kappa^{2}}
$$


Therefore in order for the solution to exist, the condition is $f_{0}<0$. In (42), $\varphi_{0}$ can be arbitrary. Hence, the Hubble rate $H=H_{0}$ might be determined by an initial condition.

In [27], the stability of the above (and related) solutions was checked and it was found that the case corresponding to phantom cosmology with $h_{0}<0$ is always stable but the case corresponding to non-phantom cosmology with $h_{0}>0$ is always unstable. Also in [31], by taking into account the higher-order string corrections to Einstein-Hilbert action, the evolution of (phantom) dark energy universe was studied. When fixed dilaton, while the presence of a cosmological constant gives stable de-Sitter fixed points in the cases of heterotic and bosonic strings, no stable de-Sitter solutions exist when a phantom fluid is present. It was found that the universe can exhibit a Big Crunch singularity with a finite time for type II string, whereas it reaches a Big Rip singularity for heterotic and bosonic strings. Thus the fate of dark energy universe crucially depends upon the type of string theory under consideration. Furthermore, a barotropic perfect fluid coupled to the scalar field (dilaton or compactification modulus) was also considered and phase space analysis and the stability of asymptotic solutions are performed for a number of models which include $(i)$ fixed scalar field, (ii) linear dilaton in string frame, and (iii) logarithmic modulus in Einstein frame. It was also shown that dilaton Gauss-Bonnet gravity (with no matter) may not explain the current acceleration of the universe. It was also studied the future evolution of the universe using the GB parametrization and found that Big Rip singularities can be avoided even in the presence of a phantom fluid because of the balance between the fluid and curvature corrections. A non-minimal coupling between the fluid and the modulus field also opens up the interesting possibility to avoid Big Rip regardless of the details of the fluid equation of state. Note also that above action has interesting applications in early time string cosmology (for recent discussion and list of references, see [32]). Definitely, dilaton Gauss-Bonnet gravity (perhaps, also with account of higher order terms like Euler invariant) deserves very deep investigation as possible candidate for dark energy.

\section{MODIFIED GRAVITY: NON-LINEAR COUPLING, COSMIC ACCELERATION AND HIERARCHY PROBLEM}

\section{A. Gravitational solution of coincidence problem}

It is interesting to investigate the role of non-minimal coupling of modified gravity with dark energy to cosmic acceleration epoch. The corresponding, quite general theory has been suggested in ref. 33] (see also [34]). As an example of such theory, the following action is considered:

$$
S=\int d^{4} x \sqrt{-g}\left\{\frac{1}{\kappa^{2}} R+\left(\frac{R}{\mu^{2}}\right)^{\alpha} L_{d}\right\} .
$$

Here $L_{d}$ is matter-like action (dark energy). The choice of parameter $\mu$ may keep away the unwanted instabilities which often occur in higher derivative theories. The second term in above action describes the non-linear coupling of matter with gravity (in parallel with $R \phi^{2}$ term which is usually required by renormalizability condition [35]). Similarly, such term may be induced by quantum effects as non-local effective action. Hence, it is natural to consider that it belongs to matter sector. (Standard matter is not included for simplicity).

By the variation over $g_{\mu \nu}$, the equation of motion follows:

$$
0=\frac{1}{\sqrt{-g}} \frac{\delta S}{\delta g_{\mu \nu}}=\frac{1}{\kappa^{2}}\left\{\frac{1}{2} g^{\mu \nu} R-R^{\mu \nu}\right\}+\tilde{T}^{\mu \nu} .
$$

Here the effective EMT tensor $\tilde{T}_{\mu \nu}$ is defined by

$$
\begin{aligned}
\tilde{T}^{\mu \nu} & \equiv \frac{1}{\mu^{2 \alpha}}\left\{-\alpha R^{\alpha-1} R^{\mu \nu} L_{d}+\alpha\left(\nabla^{\mu} \nabla^{\nu}-g^{\mu \nu} \nabla^{2}\right)\left(R^{\alpha-1} L_{d}\right)+R^{\alpha} T^{\mu \nu}\right\}, \\
T^{\mu \nu} & \equiv \frac{1}{\sqrt{-g}} \frac{\delta}{\delta g_{\mu \nu}}\left(\int d^{4} x \sqrt{-g} L_{d}\right)
\end{aligned}
$$

Let free massless scalar be a matter

$$
L_{d}=-\frac{1}{2} g^{\mu \nu} \partial_{\mu} \phi \partial_{\nu} \phi
$$

Then the equation given by the variation over $\phi$ has the following form:

$$
0=\frac{1}{\sqrt{-g}} \frac{\delta S}{\delta \phi}=\frac{1}{\sqrt{-g}} \partial_{\mu}\left(R^{\alpha} \sqrt{-g} g^{\mu \nu} \partial_{\nu} \phi\right)
$$


The metric again corresponds to FRW universe with flat 3 -space. If we assume $\phi$ only depends on $t(\phi=\phi(t))$, the solution of scalar field equation (47) is given by

$$
\dot{\phi}=q a^{-3} R^{-\alpha}
$$

Here $q$ is a constant of the integration. Hence $R^{\alpha} L_{d}=\frac{q^{2}}{2 a^{6} R^{\alpha}}$, which becomes dominant when $R$ is small (large) compared with the Einstein term $\frac{1}{\kappa^{2}} R$ if $\alpha>-1(\alpha<-1)$. Thus, one arrives at the remarkable possibility 33. that dark energy grows to asymptotic dominance over the usual matter with decrease of the curvature. At current universe, this solves the coincidence problem (the equality of the energy density for dark energy and for matter) simply by the fact of the universe expansion.

Substituting (48) into (44), the $(\mu, \nu)=(t, t)$ component of equation of motion has the following form:

$$
\begin{aligned}
0= & -\frac{3}{\kappa^{2}} H^{2}+\frac{36 q^{2}}{\mu^{2 \alpha} a^{6}\left(6 \dot{H}+12 H^{2}\right)^{\alpha+2}}\left\{\frac{\alpha(\alpha+1)}{4} \ddot{H} H+\frac{\alpha+1}{4} \dot{H}^{2}\right. \\
& \left.+\left(1+\frac{13}{4} \alpha+\alpha^{2}\right) \dot{H} H^{2}+\left(1+\frac{7}{2} \alpha\right) H^{4}\right\} .
\end{aligned}
$$

The accelerated FRW solution of (49) exists [33]

$$
a=a_{0} t^{\frac{\alpha+1}{3}} \quad\left(H=\frac{\alpha+1}{3 t}\right), \quad a_{0}^{6} \equiv \frac{\kappa^{2} q^{2}(2 \alpha-1)(\alpha-1)}{\mu^{2 \alpha} 3(\alpha+1)^{\alpha+1}\left(\frac{2}{3}(2 \alpha-1)\right)^{\alpha+2}} .
$$

Eq.(50) tells that the universe accelerates, that is, $\ddot{a}>0$ if $\alpha>2$. If $\alpha<-1$, the solution (50) describes shrinking universe if $t>0$. If the time is shifted as $t \rightarrow t-t_{s}\left(t_{s}\right.$ is a constant), the accelerating and expanding universe occurs when $t<t_{s}$. In the solution with $\alpha<-1$ there appears a Big Rip singularity at $t=t_{s}$. For the matter with the relation $p=w \rho$, where $p$ is the pressure and $\rho$ is the energy density, from the usual FRW equation, one has $a \propto t^{\frac{2}{3(w+1)}}$. For $a \propto t^{h_{0}}$ it follows $w=-1+\frac{2}{3 h_{0}}$, and the accelerating expansion $\left(h_{0}>1\right)$ of the universe occurs if $-1<w<-\frac{1}{3}$. For the case of (50), one finds

$$
w=\frac{1-\alpha}{1+\alpha} .
$$

Then if $\alpha<-1$, we have $w<-1$, which is an effective phantom. For the general matter with the relation $p=w \rho$ with constant $w$, the energy $E$ and the energy density $\rho$ behave as $E \sim a^{-3 w}$ and $\rho \sim a^{-3(w+1)}$. Thus, for the standard phantom with $w<-1$, the density becomes large with time and might generate the Big Rip. Hence, it is demonstrated that non-linear coupling of gravity with matter may produce the accelerated cosmology with the effective EoS parameter being close (above, equal or below) to -1. More complicated couplings [33] may be considered in the same fashion.

\section{B. Dynamical cosmological constant theory: an exact example}

There are many proposals to solve the cosmological constant problem dynamically. One of the possible solutions of the cosmological constant problem is pointed out by Mukohyama and Randall 36] (see also [37]). In 36], the following action similar to the one under consideration has been proposed:

$$
I=\int d^{4} x \sqrt{-g}\left[\frac{R}{2 \kappa^{2}}+\alpha_{0} R^{2}+\frac{\left(\kappa^{4} \partial_{\mu} \varphi \partial^{\mu} \varphi\right)^{q}}{2 q \kappa^{4} f(R)^{2 q-1}}-V(\varphi)\right],
$$

where $f(R)$ is a proper function. When the curvature is small, it is assumed $f(R)$ behaves as

$$
f(R) \sim\left(\kappa^{2} R^{2}\right)^{m}
$$

Here $m$ is positive. When the curvature is small, the vacuum energy, and therefore the value of the potential becomes small. Then one may assume, for the small curvature, $V(\varphi)$ behaves as

$$
V(\varphi) \sim V_{0}\left(\varphi-\varphi_{c}\right)
$$


Here $V_{0}$ and $\varphi_{c}$ are constants. If $q>1 / 2$, the factor in front of the kinetic term of $\varphi$ in (52) becomes large. This makes the time development of the scalar field $\varphi$ very slow and it is expected that $\varphi$ does not reach $\varphi_{c}$. This model may explain the acceleration of the present universe. The model (52) is also expected to be stable under the radiative corrections. In fact, when the curvature is small and the time development of the curvature can be neglected, if we rescale the scalar field $\varphi$ as $\varphi \rightarrow R^{m(2 q-1) / q} \varphi$, the curvature in the kinetic term can be absorbed into the redefinition of $\varphi$ and there appear factors including $R^{m(2 q-1) / q}$. Therefore if $m(2 q-1) / q>0$, the interactions could be suppressed when the curvature is small and there will not appear the radiative correction to the vacuum energy except the one-loop corrections.

There is an exactly solvable model [38], which realizes the above scenario. Let us choose

$$
f(R)=\beta R^{2}, \quad V(\varphi)=V_{0}\left(\varphi-\varphi_{c}\right) .
$$

Here $\beta$ is a constant. $R^{2}$ term is neglected by putting $\alpha_{0}=0$ in (52) since the curvature is small. Searching for the solution (6) in sect [1] and choosing $\varphi=\varphi_{c}+\varphi_{0} / t^{2}$ or $\varphi=\varphi_{c}+\varphi_{0} /\left(t_{s}-t\right)^{2}$, the following restrictions are obtained [38]

$$
\varphi_{0}^{2}=\frac{54 \beta\left(-1+2 h_{0}\right)^{3} h_{0}^{4}}{\kappa^{2}\left(12 h_{0}^{2}-2 h_{0}-1\right)}, \quad V_{0}= \pm \frac{3 h_{0}+1}{\sqrt{6 \kappa^{2}\left(12 h_{0}^{2}-2 h_{0}-1\right)\left(-1+2 h_{0}\right)}}
$$

Since $\varphi_{0}^{2}$ should be positive, one finds

$$
\begin{array}{ll}
\text { when } \beta>0, & \frac{1-\sqrt{13}}{12}<h_{0}<\frac{1+\sqrt{13}}{12} \text { or } h_{0} \geq \frac{1}{2}, \\
\text { when } \beta<0, & h_{0}<\frac{1-\sqrt{13}}{12} \text { or } \frac{1+\sqrt{13}}{12}<h_{0} \leq \frac{1}{2} .
\end{array}
$$

For example, if $h_{0}=-1 / 60$, which gives $w_{\text {eff }}=-1.025$, we find

$$
\kappa V_{0}= \pm \frac{19}{34} \sqrt{\frac{15}{31}}= \pm 0.388722 \ldots
$$

It is interesting that the value of $w_{\text {eff }}$ is consistent with the observed bounds. (Note that recently the above model has been reconsidered in ref. [39]).

For $h_{0}>0$ case, since $R=6 \dot{H}+12 H^{2}$, the curvature $R$ decreases as $t^{-2}$ with time $t$ and $\varphi$ approaches to $\varphi_{c}$ but does not arrive at $\varphi_{c}$ in a finite time, as expected in [36].

As $H$ behaves as $h_{0} / t$ or $h_{0} /\left(t_{s}-t\right)$ for [6]) in sect [I] if we substitute the value of the age of the present universe $10^{10}$ years $\sim\left(10^{-33} \mathrm{eV}\right)^{-1}$ into $t$ or $t_{s}-t$, the observed value of $H$ could be reproduced, which could explain the smallness of the effective cosmological constant $\Lambda \sim H^{2}$. Note that even if there is no potential term, that is, $V_{0}=0$, when $\beta<0$, there is a solution

$$
h_{0}=-\frac{1}{3}<\frac{1-\sqrt{13}}{12}=-0.2171 \ldots,
$$

which gives the EoS parameter : $w=-3$, although $w$ is not realistic. Playing with different choices of the potential and non-linear coupling more realistic predictions may be obtained.

\section{Hierarchy problem in modified gravity}

Hierarchy problem is known to be the fundamental one in high energy physics. It is interesting to understand if modified gravity approach may help in resolution of hierarchy problem? Recently the hierarchy problem has been investigated, in [40], in frames of scalar-tensor theory. Here, similar proposal will be discussed in the modified gravity. In [40], in order to generate the hierarchy, a small scale, which is the vacuum decay rate $\Gamma_{v a c}$, was considered. Instead of $\Gamma_{v a c}$, we here use the age of the universe $\sim 10^{-33} \mathrm{eV}$, as the small mass scale.

We now start from the action for modified gravity coupled with matter (II) in sect III Let us assume that the matter Lagrangian density $\mathcal{L}_{\text {matter }}$ contains a Higgs-like scalar field $\varphi$

$$
\mathcal{L}_{\text {matter }}=-\frac{1}{2} \nabla^{\mu} \varphi \nabla_{\mu} \varphi+\frac{\mu^{2}}{2} \varphi^{2}-\lambda \varphi^{4}+\cdots .
$$


Under the conformal transformation $g_{\mu \nu} \rightarrow \mathrm{e}^{\sigma} g_{\mu \nu}$, the matter Lagrangian density $\mathcal{L}_{\text {matter }}$ is transformed as

$$
\mathcal{L}_{\text {matter }} \rightarrow \mathcal{L}_{\text {matter }}^{\sigma}=-\frac{\mathrm{e}^{\sigma}}{2} \nabla^{\mu} \varphi \nabla_{\mu} \varphi+\frac{\mu^{2} \mathrm{e}^{2 \sigma}}{2} \varphi^{2}-\lambda \mathrm{e}^{2 \sigma} \varphi^{4}+\cdots
$$

By redefining $\varphi$ as $\varphi \rightarrow \mathrm{e}^{-\sigma / 2} \varphi, \mathcal{L}_{\text {matter }}^{\sigma}$ acquires the following form

$$
\mathcal{L}_{\text {matter }}^{\sigma} \sim-\frac{1}{2} \nabla^{\mu} \varphi \nabla_{\mu} \varphi+\frac{\mu^{2} \mathrm{e}^{\sigma}}{2} \varphi^{2}-\lambda \varphi^{4}+\cdots
$$

where the time derivative of $\sigma$ is neglected. Then, the massive parameter $\mu$, which determines the weak scale, is effectively transformed as $\mu \rightarrow \tilde{\mu} \equiv \mathrm{e}^{\sigma / 2} \mu$. In principle, $\mu$ can be of the order of the Planck scale $10^{19} \mathrm{GeV}$, but if $\mathrm{e}^{\sigma / 2} \sim 10^{-17}$ in the present universe, $\tilde{\mu}$ could be of the order of $10^{2} \mathrm{GeV}$, which is the scale of the weak interaction. Therefore there is a quite natural possibility that the hierarchy problem can be solved by above version of modified gravity (similar solution of hierarchy problem may be found in two scalar-tensor gravity of ref. 41] as it has been shown in [42].)

One may consider the model [42]:

$$
f(R)=R+f_{0} R^{\alpha}
$$

with constant $f_{0}$ and $\alpha$. (Without the first term the restrictions to the parameter $\alpha$ have been recently studied in ref. 43].) If $\alpha<1$, the second term dominates, when the curvature is small. Assuming that the EoS parameter, $w$, of matter is constant one gets [14] the accelerated FRW cosmology solution with the effective EoS parameter $w_{\text {eff }}$ (23).

Neglecting the first term in (63), it follows

$$
\mathrm{e}^{\sigma / 2} \sim \frac{1}{\sqrt{f_{0} \alpha R^{\alpha-1}}} .
$$

In the present universe, $R \sim\left(10^{-33} \mathrm{eV}\right)^{2}$. Assume now that $f_{0}$ is of the order of the Planck scale $\sim 10^{19} \mathrm{GeV}=10^{28} \mathrm{eV}$ as $f_{0} \sim\left(10^{28} \mathrm{eV}\right)^{\frac{1}{2(\alpha-1)}}$. Then, Eq. (64) yields

$$
\mathrm{e}^{\sigma / 2} \sim 10^{61(\alpha-1)}
$$

If we furthermore assume that $-17=61(\alpha-1)$, we find $\alpha=44 / 61$. In that case, if $w>-1$, weff $>-1$ and the universe is not in its phantom phase, but (65) hints towards the possibility that modified gravity can solve the hierarchy problem.

Let us write the action of the scalar-tensor theory as

$$
\begin{aligned}
S & \left.=\frac{1}{\kappa^{2}} \int d^{4} x \sqrt{-g} \mathrm{e}^{\alpha \phi}\left(R-\frac{1}{2} \partial_{\mu} \phi \partial^{\mu} \phi-V_{0} \mathrm{e}^{-2 \phi / \phi_{0}}\right)\right)+\int d^{4} x \sqrt{-g} \mathcal{L}_{\text {matter }}, \\
\mathcal{L}_{\text {matter }} & =-\frac{1}{2} \partial^{\mu} \varphi \partial_{\mu} \varphi+\frac{\mu^{2}}{2} \varphi^{2}-\lambda \varphi^{4}+\cdots .
\end{aligned}
$$

This action could be regarded as the Jordan frame action (see sect 1 ). Scalar field $\varphi$ could be identified with the Higgs field in the weak(-electromagnetic) interaction. Now the ratio of the inverse of the the effective gravitational coupling $\tilde{\kappa}=\kappa \mathrm{e}^{-\frac{\alpha \phi}{2}}$ and the Higgs mass $\mu$ is given by

$$
\frac{\frac{1}{\tilde{\kappa}}}{\mu}=\frac{\mathrm{e}^{\frac{\alpha \phi}{2}}}{\kappa \mu} .
$$

Hence, even if both of $1 / \kappa$ and $\mu$ are of the order of the weak interaction scale, if $\mathrm{e}^{\frac{\alpha \phi}{2}} \sim 10^{17}, 1 / \tilde{\kappa}$ could have an order of the Planck scale.

By rescaling the metric and the Higgs scalar $\varphi$ as

$$
g_{\mu \nu} \rightarrow \mathrm{e}^{-\alpha \phi} g_{\mu \nu}, \quad \varphi \rightarrow \mathrm{e}^{\frac{\alpha \phi}{2}} \varphi,
$$

the Einstein frame action is obtained:

$$
\begin{aligned}
S= & \left.\frac{1}{\kappa^{2}} \int d^{4} x \sqrt{-g}\left(R-\frac{1}{2}\left(1+3 \alpha^{2}\right) \partial_{\mu} \phi \partial^{\mu} \phi-V_{0} \mathrm{e}^{-2 \phi\left(1 / \phi_{0}+\alpha\right)}\right)\right) \\
& +\int d^{4} x \sqrt{-g} \mathcal{L}_{\text {matter }} \\
\mathcal{L}_{\text {matter }}= & -\frac{1}{2}\left(\partial^{\mu} \varphi+\frac{\alpha}{2} \partial^{\mu} \phi \varphi\right)\left(\partial_{\mu} \varphi+\frac{\alpha}{2} \partial_{\mu} \phi \varphi\right)+\frac{\mu^{2} \mathrm{e}^{-\alpha \phi}}{2} \varphi^{2}-\lambda \varphi^{4}+\cdots
\end{aligned}
$$


In the Einstein frame, the gravitational coupling $\kappa$ is constant but the effective Higgs mass $\tilde{\mu}$ defined by $\tilde{\mu} \equiv \mathrm{e}^{-\frac{\alpha \phi}{2}} \mu$ can be time-dependent. Hence, the ratio of $1 / \kappa$ and $\tilde{\mu}$ is given by

$$
\frac{\frac{1}{\kappa}}{\tilde{\mu}}=\frac{\mathrm{e}^{\frac{\alpha \phi}{2}}}{\kappa \mu}
$$

which is identical with (67). Hence, even if both of $1 / \kappa$ and $\mu$ are of the order of the Planck scale, if $\mathrm{e}^{\frac{\alpha \phi}{2}} \sim 10^{17}, \tilde{\mu}$ could have an order of the weak interaction scale. Therefore the solution of the hierarchy problem does not essentially depend on the frame choice.

Nevertheless, note that the cosmological time variables in two frames could be different due to the scale transformation (68) as $d t \rightarrow d \tilde{t}=\mathrm{e}^{-\frac{\alpha \phi}{2}} d t$. Therefore the time-intervals are different in the two frames. The units of time and the length are now defined by the electromagnetism. Then the frame where the electromagnetic fields do not couple with the scalar field $\phi$ could be physically more preferrable. Since the electromagnetic interaction is a part of the electro-weak interaction, the Jordan frame in (66) could be more preferrable from the point of view of the solution of hierarchy problem.

\section{LATE-TIME COSMOLOGY IN MODIFIED GAUSS-BONNET GRAVITY}

\section{A. $f(G)$ gravity}

Our next example is modified Gauss-Bonnet gravity. Let us start from the action [42, 44]:

$$
S=\int d^{4} x \sqrt{-g}\left(\frac{1}{2 \kappa^{2}} R+f(G)+\mathcal{L}_{m}\right) .
$$

Here $\mathcal{L}_{m}$ is the matter Lagrangian density and $G$ is the GB invariant: $G=R^{2}-4 R_{\mu \nu} R^{\mu \nu}+R_{\mu \nu \xi \sigma} R^{\mu \nu \xi \sigma}$. By variation over $g_{\mu \nu}$ one gets:

$$
\begin{aligned}
& 0=\frac{1}{2 \kappa^{2}}\left(-R^{\mu \nu}+\frac{1}{2} g^{\mu \nu} R\right)+T^{\mu \nu}+\frac{1}{2} g^{\mu \nu} f(G)-2 f^{\prime}(G) R R^{\mu \nu} \\
& +4 f^{\prime}(G) R_{\rho}^{\mu} R^{\nu \rho}-2 f^{\prime}(G) R^{\mu \rho \sigma \tau} R_{\rho \sigma \tau}^{\nu}-4 f^{\prime}(G) R^{\mu \rho \sigma \nu} R_{\rho \sigma}+2\left(\nabla^{\mu} \nabla^{\nu} f^{\prime}(G)\right) R \\
& -2 g^{\mu \nu}\left(\nabla^{2} f^{\prime}(G)\right) R-4\left(\nabla_{\rho} \nabla^{\mu} f^{\prime}(G)\right) R^{\nu \rho}-4\left(\nabla_{\rho} \nabla^{\nu} f^{\prime}(G)\right) R^{\mu \rho} \\
& +4\left(\nabla^{2} f^{\prime}(G)\right) R^{\mu \nu}+4 g^{\mu \nu}\left(\nabla_{\rho} \nabla_{\sigma} f^{\prime}(G)\right) R^{\rho \sigma}-4\left(\nabla_{\rho} \nabla_{\sigma} f^{\prime}(G)\right) R^{\mu \rho \nu \sigma},
\end{aligned}
$$

where $T^{\mu \nu}$ is the matter EM tensor. By choosing the spatially-flat FRW universe metric (4) in sect II the equation corresponding to the first FRW equation has the following form:

$$
0=-\frac{3}{\kappa^{2}} H^{2}+G f^{\prime}(G)-f(G)-24 \dot{G} f^{\prime \prime}(G) H^{3}+\rho_{m}
$$

where $\rho_{m}$ is the matter energy density. When $\rho_{m}=0$, Eq. (73) has a deSitter universe solution where $H$, and therefore $G$, are constant. For $H=H_{0}$, with constant $H_{0}$, Eq. (173) turns into

$$
0=-\frac{3}{\kappa^{2}} H_{0}^{2}+24 H_{0}^{4} f^{\prime}\left(24 H_{0}^{4}\right)-f\left(24 H_{0}^{4}\right)
$$

For a large number of choices of the function $f(G)$, Eq. (74) has a non-trivial $\left(H_{0} \neq 0\right)$ real solution for $H_{0}($ deSitter universe). The late-time cosmology for above theory without matter has been discussed for a number of examples in refs. 42, 44]. We follow these works here.

We now consider the case $\rho_{m} \neq 0$. Assuming that the EoS parameter $w \equiv p_{m} / \rho_{m}$ for matter $\left(p_{m}\right.$ is the pressure of matter) is a constant then, by using the conservation of energy: $\dot{\rho}_{m}+3 H\left(\rho_{m}+p_{m}\right)=0$, we find $\rho=\rho_{0} a^{-3(1+w)}$. The function $f(G)$ is chosen as

$$
f(G)=f_{0}|G|^{\beta}
$$

with constant $f_{0}$ and $\beta$. If $\beta<1 / 2, f(G)$ term becomes dominant compared with the Einstein term when the curvature is small. If we neglect the contribution from the Einstein term in (73), assuming FRW anzats (6) in sect [I] the following solution may be found

$$
h_{0}=\frac{4 \beta}{3(1+w)}, \quad a_{0}=\left[-\frac{f_{0}(\beta-1)}{\left(h_{0}-1\right) \rho_{0}}\left\{24\left|h_{0}^{3}\left(-1+h_{0}\right)\right|\right\}^{\beta}\left(h_{0}-1+4 \beta\right)\right]^{-\frac{1}{3(1+w)}} .
$$


Then the effective EoS parameter $w_{\text {eff }}$ (11) in sect is less than -1 if $\beta<0$, and for $w>-1$ is

$$
w_{\text {eff }}=-1+\frac{2}{3 h_{0}}=-1+\frac{1+w}{2 \beta},
$$

which is again less than -1 for $\beta<0$. Thus, if $\beta<0$, we obtain an effective phantom with negative $h_{0}$ even in the case when $w>-1$. In the phantom phase, there might seem to occur the Big Rip at $t=t_{s}[15$. Near this Big Rip , however, the curvature becomes dominant and then the Einstein term dominates, so that the $f(G)$ term can be neglected. Therefore, the universe behaves as $a=a_{0} t^{2 / 3(w+1)}$ and as a consequence the Big Rip does not eventually occur. The phantom era is transient.

The case when $0<\beta<1 / 2$ may be also considered. As $\beta$ is positive, the universe does not reach here the phantom phase. When the curvature is strong, the $f(G)$ term in the action (71) can be neglected and we can work with Einstein gravity. Then if $w$ is positive, the matter energy density $\rho_{m}$ should behave as $\rho_{m} \sim t^{-2}$, but $f(G)$ goes as $f(G) \sim t^{-4 \beta}$. Then, for late times (large $t$ ), the $f(G)$ term may become dominant as compared with the matter one. If we neglect the contribution from matter, Eq. (73) has a deSitter universe solution where $H$, and therefore $G$, are constant. If $H=H_{0}$ with constant $H_{0}$, Eq. (73) looks as (74). As a consequence, even if we start from the deceleration phase with $w>-1 / 3$, we may also reach an asymptotically deSitter universe, which is an accelerated universe. Correspondingly, also here there could be a transition from acceleration to deceleration of the universe.

Now, we consider the case when the contributions coming from the Einstein and matter terms can be neglected. Then, Eq. (73) reduces to

$$
0=G f^{\prime}(G)-f(G)-24 \dot{G} f^{\prime \prime}(G) H^{3}
$$

If $f(G)$ behaves as (75), from assumption (6) in sect [II we obtain

$$
0=(\beta-1) h_{0}^{6}\left(h_{0}-1\right)\left(h_{0}-1+4 \beta\right) .
$$

As $h_{0}=1$ implies $G=0$, one may choose

$$
h_{0}=1-4 \beta \text {, }
$$

and Eq. (11) in sect gives

$$
w_{\mathrm{eff}}=-1+\frac{2}{3(1-4 \beta)} .
$$

Therefore, if $\beta>0$, the universe is accelerating $\left(w_{\text {eff }}<-1 / 3\right)$ and if $\beta>1 / 4$, the universe is in a phantom phase $\left(w_{\text {eff }}<-1\right)$. Thus, we are led to consider the following model:

$$
f(G)=f_{i}|G|^{\beta_{i}}+f_{l}|G|^{\beta_{l}}
$$

where we assume that

$$
\beta_{i}>\frac{1}{2}, \quad \frac{1}{2}>\beta_{l}>\frac{1}{4}
$$

Then, when the curvature is large, as in the primordial universe, the first term dominates, compared with the second one and the Einstein term, and gives

$$
-1>w_{\mathrm{eff}}=-1+\frac{2}{3\left(1-4 \beta_{i}\right)}>-5 / 3 .
$$

On the other hand, when the curvature is small, as is the case in the present universe, the second term in (82) dominates, compared with the first one and the Einstein term, and yields

$$
w_{\text {eff }}=-1+\frac{2}{3\left(1-4 \beta_{l}\right)}<-5 / 3 .
$$

Therefore, theory (82) can in fact produce a model which is able to describe inflation and the late-time acceleration of the universe in the unified way.

Instead of (83), one may also choose $\beta_{l}$ as

$$
\frac{1}{4}>\beta_{l}>0
$$


which gives

$$
-\frac{1}{3}>w_{\mathrm{eff}}>-1 .
$$

Then, what we obtain is an effective quintessence. Moreover, by properly adjusting the couplings $f_{i}$ and $f_{l}$ in (82), one can obtain a period where the Einstein term dominates and the universe is in a deceleration phase. After that, there would come a transition from deceleration to acceleration, when the GB term becomes the dominant one. More choices of $f(G)$ may be studied with the purpose of the construction of natural accelerating universes.

Let us address the issue of the correction to Newton law. Let $g_{(0)}$ be a solution of (72) and represent the perturbation of the metric as $g_{\mu \nu}=g_{(0) \mu \nu}+h_{\mu \nu}$. First, we consider the perturbation around the deSitter background which is a solution of (74). We write the deSitter space metric as $g_{(0) \mu \nu}$, which gives the following Riemann tensor:

$$
R_{(0) \mu \nu \rho \sigma}=H_{0}^{2}\left(g_{(0) \mu \rho} g_{(0) \nu \sigma}-g_{(0) \mu \sigma} g_{(0) \nu \rho}\right)
$$

The flat background corresponds to the limit of $H_{0} \rightarrow 0$. For simplicity, the following gauge condition is chosen: $g_{(0)}^{\mu \nu} h_{\mu \nu}=\nabla_{(0)}^{\mu} h_{\mu \nu}=0$. Then Eq. (72) gives

$$
0=\frac{1}{4 \kappa^{2}}\left(\nabla^{2} h_{\mu \nu}-2 H_{0}^{2} h_{\mu \nu}\right)+T_{\mu \nu} .
$$

The GB term contribution does not appear except in the length parameter $1 / H_{0}$ of the deSitter space, which is determined with account to the GB term. This may occur due to the special structure of GB invariant. Eq. (89) tells us that there is no correction to Newton's law in deSitter and even in the flat background corresponding to $H_{0} \rightarrow 0$, whatever is the form of $f$ (at least, with above gauge condition). Note that the study of newtonian limit in $1 / R$ gravity (where significant corrections to newton law may appear) and its extensions has been done in [4, 7]. For most $1 / R$ models the corrections to Newton law do not comply with solar system tests.

By introducing two auxilliary fields, $A$ and $B$, one can rewrite the action (71) as

$$
\begin{aligned}
S= & \int d^{4} x \sqrt{-g}\left(\frac{1}{2 \kappa^{2}} R+B(G-A)\right. \\
& \left.+f(A)+\mathcal{L}_{m}\right) .
\end{aligned}
$$

Varying over $B$, it follows that $A=G$. Using this in (90), the action (71) is recovered. On the other hand, varying over $A$ in (90), one gets $B=f^{\prime}(A)$, and hence

$$
S=\int d^{4} x \sqrt{-g}\left(\frac{1}{2 \kappa^{2}} R+f^{\prime}(A) G-A f^{\prime}(A)+f(A)\right) .
$$

By varying over $A$, the relation $A=G$ is obtained again. The scalar is not dynamical as it has no kinetic term. We may add, however, a kinetic term to the action by hand

$$
S=\int d^{4} x \sqrt{-g}\left(\frac{1}{2 \kappa^{2}} R-\frac{\epsilon}{2} \partial_{\mu} A \partial^{\mu} A+f^{\prime}(A) G-A f^{\prime}(A)+f(A)\right) .
$$

Then one obtains a dynamical scalar theory coupled with the Gauss-Bonnet invariant and with a potential. It is known that a theory of this kind has no ghosts and it is stable, in general. Actually, it is related with string-inspired dilaton gravity proposed as alternative for dark energy [27, 31]. Then, in case that the limit $\epsilon \rightarrow 0$ can be obtained smoothly, the corresponding $f(G)$ theory has no ghost and could actually be stable. It may be of interest to study the cosmology of such theory in the limit $\epsilon \rightarrow 0$.

\section{B. $f(G, R)$ gravity}

It is interesting to study late-time cosmology in generalized theories, which include both the functional dependence from curvature as well as from the Gauss-Bonnet term [42]:

$$
S=\int d^{4} x \sqrt{-g}\left(f(G, R)+\mathcal{L}_{m}\right)
$$


The following solvable model is considered:

$$
f(G, R)=R \tilde{f}\left(\frac{G}{R^{2}}\right), \quad \tilde{f}\left(\frac{G}{R^{2}}\right)=\frac{1}{2 \kappa^{2}}+f_{0}\left(\frac{G}{R^{2}}\right) .
$$

The FRW solution may be found again:

$$
H=\frac{h_{0}}{t}, \quad h_{0}=\frac{\frac{3}{\kappa^{2}}-2 f_{0} \pm \sqrt{8 f_{0}\left(f_{0}-\frac{3}{8 \kappa^{2}}\right)}}{\frac{6}{\kappa^{2}}+2 f_{0}} .
$$

Then, for example, if $\kappa^{2} f_{0}<-3$, there is a solution describing a phantom with $h_{0}<-1-\sqrt{2}$ and a solution describing the effective matter with $h_{0}>-1+\sqrt{2}$. Late-time cosmology in other versions of such theory may be constructed.

\section{INHOMOGENEOUS EQUATION OF STATE OF THE UNIVERSE DARK FLUID}

Let us remind several simple facts about the universe filled with ideal fluid. By using the energy conservation law $0=\dot{\rho}+3 H(p+\rho)$, when $\rho$ and $p$ satisfy the following simple $\operatorname{EOS} p=w \rho$ with constant $w$, we find $\rho=\rho_{0} a^{-3(1+w)}$. Then by using the first FRW equation $\left(3 / \kappa^{2}\right) H^{2}=\rho$, the well-known solution follows $a=a_{0}\left(t-t_{1}\right)^{\frac{2}{3(w+1)}}(w>-1)$ or $a_{0}\left(t_{2}-t\right)^{\frac{2}{3(w+1)}} w \neq-1(w<-1)$ and $a=a_{0} \mathrm{e}^{\kappa t \sqrt{\frac{\rho_{0}}{3}}}$ when $w=-1$, which is the deSitter universe. Here $t_{1}$ and $t_{2}$ are constants of the integration. When $w<-1$, there appears a Big Rip singularity in a finite time at $t=t_{2}$.

In general, the singularities in dark energy universe may behave in a different way. One may classify the future singularities as follows [18]:

- Type I ("Big Rip") : For $t \rightarrow t_{s}, a \rightarrow \infty, \rho \rightarrow \infty$ and $|p| \rightarrow \infty$

- Type II ("sudden") : For $t \rightarrow t_{s}, a \rightarrow a_{s}, \rho \rightarrow \rho_{s}$ or 0 and $|p| \rightarrow \infty$

- Type III : For $t \rightarrow t_{s}, a \rightarrow a_{s}, \rho \rightarrow \infty$ and $|p| \rightarrow \infty$

- Type IV : For $t \rightarrow t_{s}, a \rightarrow a_{s}, \rho \rightarrow 0,|p| \rightarrow 0$ and higher derivatives of $H$ diverge. This also includes the case when $\rho(p)$ or both of them tend to some finite values while higher derivatives of $H$ diverge.

Here $t_{s}, a_{s}$ and $\rho_{s}$ are constants with $a_{s} \neq 0$. The type I may correspond to the Big Rip singularity, which emerges when constant $w$ is less than -1 . The type II corresponds to the sudden future singularity [16] at which $a$ and $\rho$ are finite but $p$ diverges. The type III appears for the model with $p=-\rho-A \rho^{\alpha}$ [19, 28], which is different from the sudden future singularity in the sense that $\rho$ diverges. Now, let us discuss the universe filled with ideal fluid with inhomogeneous EoS. (It is assumed that GR is valid). Effectively, this approach overlaps with modified gravity one as inhomogeneous contribution may be considered as one coming from new gravitational terms. Moreover, it may be an extremely useful in the situation when modified gravity represents highly non-linear theory where only some features (but not the whole Lagrangian) are known like is currently the case with M-theory.

\section{A. The singularities in the inhomogeneous EoS dark fluid universe}

One may start from the dark fluid with the following EOS:

$$
p=-\rho-f(\rho),
$$

where $f(\rho)$ can be an arbitrary function in general. The choice $f(\rho) \propto \rho^{\alpha}$ with a constant $\alpha$ was proposed in Ref. [28] and was investigated in detail in Ref.[18, 19]. Then the scale factor is given by

$$
a=a_{0} \exp \left(\frac{1}{3} \int \frac{d \rho}{f(\rho)}\right),
$$

and the cosmological time may be found

$$
t=\int \frac{d \rho}{\kappa \sqrt{3 \rho} f(\rho)},
$$

As an example we may consider the case that

$$
f(\rho)=A \rho^{\alpha} .
$$

Then we find [18]: 
- In case $\alpha=1 / 2$ or $\alpha=0$, there does not appear any singularity.

- In case $\alpha>1$, when $t \rightarrow t_{0}$, the energy density behaves as $\rho \rightarrow \infty$ and therefore $|p| \rightarrow \infty$. Then the scale factor $a$ is finite even if $\rho \rightarrow \infty$. Therefore $\alpha>1$ case corresponds to type III singularity.

- In $\alpha=1$ case, if $A>0$, there occurs the Big Rip or type I singularity but if $A \leq 0$, there does not appear future singularity.

- In case $1 / 2<\alpha<1$, when $t \rightarrow t_{0}$, all of $\rho,|p|$, and $a$ diverge if $A>0$ then this corresponds to type I singularity.

- In case $0<\alpha<1 / 2$, when $t \rightarrow t_{0}$, we find $\rho,|p| \rightarrow 0$ and $a \rightarrow a_{0}$ but

$$
\ln a \sim\left|t-t_{0}\right|^{\frac{\alpha-1}{\alpha-1 / 2}} .
$$

Since the exponent $(\alpha-1) /(\alpha-1 / 2)$ is not always an integer, even if $a$ is finite, the higher derivatives of $H$ diverge in general. Therefore this case corresponds to type IV singularity.

- In case $\alpha<0$, when $t \rightarrow t_{0}$, we find $\rho \rightarrow 0, a \rightarrow a_{0}$ but $|p| \rightarrow \infty$. Therefore this case corresponds to type II singularity.

Hence, the brief review of dark fluid FRW cosmology with specific homogeneous EOS as well as its late-time behaviour (singularities) is given (see [18] for more detail).

At the next step, we consider the inhomogeneous EOS for dark fluid, so that the dependence from Hubble parameter is included in EOS. This new terms may origin from string/M-theory, braneworld or modified gravity. One more motivation for such EOS comes from including of time-dependent bulk viscosity in dark fluid EOS [45] or from the modification of gravity. Hence, we suggest the following EOS [46]

$$
p=-\rho+f(\rho)+G(H) .
$$

where $G(H)$ is some function.

As an example, one may consider the case

$$
f(\rho)=A \rho^{\alpha} \rightarrow f(\rho)+G(H)=-A \rho^{\alpha}-B H^{2 \beta} .
$$

Here, it follows 46.

- In case $\alpha>1$, for most values of $\beta$, there occurs type III singularity. In addition to the type III singularity, when $0<\beta<1 / 2$, there occurs type IV singularity and when $\beta<0$, there occurs type II singularity.

- $\alpha=1$ case, if $\beta>1$, the singularity becomes type III. If $\beta<1$ and $A>0$, there occurs the Big Rip or type I singularity. In addition to the type I singularity, we have type IV singularity when $0<\beta<1 / 2$ and type II when $\beta<1$.

- In case $1 / 2<\alpha<1$, one sees singularity of type III for $\beta>1$, type I for $1 / 2 \leq \beta<1$ (even for $\beta=1 / 2$ ) or $\beta=1$ and $B^{\prime}>0(B>0)$ case. In addition to type I, type IV case occurs for $0<\beta<1 / 2$, and type II for $\beta<0$.

- In case $\alpha=1 / 2$, we have singularity of type III for $\beta>1$, type I for $1 / 2<\beta<1$ or $\beta=1$ and $B^{\prime}>0(B>0)$, type IV for $0<\beta<1 / 2$, and type II for $\beta<0$. When $\beta=1 / 2$ or $\beta=0$, there does not appear any singularity.

- In case $0<\alpha<1 / 2$, we find type IV for $0<\beta<1 / 2$, and type II for $\beta<0$. In addition to type IV singularity, there occurs singularity of type III for $\beta>1$, type I for $1 / 2 \leq \beta<1$ or $\beta=1$ and $B^{\prime}>0(B>0)$ case.

- In case $\alpha<0$, there will always occur type II singularity. In addition to type II singularity, we have a singularity of type III for $\beta>1$, type I for $1 / 2 \leq \beta<1$ or $\beta=1$ and $B^{\prime}>0(B>0)$ case.

Thus, we demonstrated how the modification of EOS by Hubble dependent, inhomogeneous term changes the structure of singularities in late-time dark energy universe.

In general, EOS needs to be double-valued in order for the transition (crossing of phantom divide) to occur between the region $w<-1$ and the region $w>-1$. Then there could not be one-to-one correspondence between $p$ and $\rho$. In such a case, instead of (101), we may suggest the implicit, inhomogeneous equation of the state

$$
F(p, \rho, H)=0 \text {. }
$$


The following example may be of interest:

$$
(p+\rho)^{2}-C_{0} \rho^{2}\left(1-\frac{H_{0}}{H}\right)=0 .
$$

Here $C_{0}$ and $H_{0}$ are positive constants. Hence, the Hubble rate looks as

$$
H=\frac{16}{9 C_{0}^{2} H_{0}\left(t-t_{-}\right)\left(t_{+}-t\right)} .
$$

and

$$
p=-\rho\left\{1+\frac{3 C_{0}^{2}}{4 H_{0}}\left(t-t_{0}\right)\right\}, \quad \rho=\frac{2^{8}}{3^{3} C_{0}^{4} H_{0}^{2} \kappa^{2}\left(t-t_{-}\right)^{2}\left(t_{+}-t\right)^{2}} .
$$

In (105), since $t_{-}<t_{0}<t_{+}$, as long as $t_{-}<t<t_{+}$, the Hubble rate $H$ is positive. The Hubble rate $H$ has a minimum $H=H_{0}$ when $t=t_{0}=\left(t_{-}+t_{+}\right) / 2$ and diverges when $t \rightarrow t_{ \pm}$. Then one may regard $t \rightarrow t_{-}$as a Big Bang singularity and $t \rightarrow t_{+}$as a Big Rip one. As clear from (106), the parameter $w=p / \rho$ is larger than -1 when $t_{-}<t<t_{0}$ and smaller than -1 when $t_{0}<t<t_{+}$. Therefore there occurs the crossing of phantom divide $w=-1$ when $t=t_{0}$ thanks to the effect of inhomogeneous term in EOS.

In principle, the more general EOS may contain the derivatives of $H$, like $\dot{H}, \ddot{H}, \ldots$ More general EOS than (103) may have the following form:

$$
F(p, \rho, H, \dot{H}, \ddot{H}, \cdots)=0
$$

Trivial example is that

$$
p=w \rho-\frac{2}{\kappa^{2}} \dot{H}-\frac{3(1+w)}{\kappa^{2}} H^{2} .
$$

By using the first and second FRW equations $\rho=\left(3 / \kappa^{2}\right) H^{2}$ and $p=-\left(2 / \kappa^{2}\right) \dot{H}-\left(3 / \kappa^{2}\right) H^{2}$, Eq. (108) becomes an identity, which means that any FRW cosmology can be a solution if EOS (108) is assumed.

\section{B. Origin of the inhomogeneous terms}

In order to give the support for an inhomogeneous EoS dark fluid [47] we will indicate how the extra terms may appear from the modifications of General Relativity. Let us start with the action of the scalar-tensor theory (25) in sect TII The energy density $\rho$ and the pressure $p$ for the scalar field $\phi$ are given by

$$
\rho=\frac{1}{2} \omega(\phi) \dot{\phi}^{2}+V(\phi), \quad p=\frac{1}{2} \omega(\phi) \dot{\phi}^{2}-V(\phi) .
$$

Since we can always redefine the scalar field $\phi$ as $\phi \rightarrow F(\phi)$ by an arbitrary function $F(\phi)$, we can choose the scalar field to be a time coordinate; $\phi=t$. Furthermore we consider the case that $\omega(\phi)$ and $V(\phi)$ are given by a single function $f(\phi)$ as (see sect

$$
\omega(\phi)=-\frac{2}{\kappa^{2}} f^{\prime}(\phi), \quad V(\phi)=\frac{1}{\kappa^{2}}\left(3 f(\phi)^{2}+f^{\prime}(\phi)\right) .
$$

Hence, one finds

$$
\rho=\frac{3}{\kappa^{2}} f(\phi)^{2}, \quad p=-\frac{3}{\kappa^{2}} f(\phi)^{2}-\frac{2}{\kappa^{2}} f^{\prime}(\phi) .
$$

Since $\rho=f^{-1}(\kappa \sqrt{\rho / 3})$, we obtain the inhomogeneous equation of the state

$$
p=-\rho-\frac{2}{\kappa^{2}} f^{\prime}\left(f^{-1}\left(\kappa \sqrt{\frac{\rho}{3}}\right)\right)
$$

As an example, we consider the case

$$
f=f_{0} \phi^{2}+f_{1}
$$


with constant $f_{0}$ and $f_{1}$. Then (112) gives

$$
0=(p+\rho)^{2}-\frac{4 f_{0}}{\kappa^{4}} f\left(1-\frac{f_{1}}{f}\right) .
$$

If the matter contribution is neglected, from the first FRW equation, one finds

$$
f^{2}=\frac{\kappa^{2}}{3} \rho=H^{2}
$$

Then we may rewrite (114) as

$$
0=(p+\rho)^{2}-\frac{4 f_{0}}{\kappa^{3}} \sqrt{\frac{\rho}{3}}\left(1-\frac{f_{1}}{H}\right),
$$

which has a structure similar to (104). One may also consider the case that

$$
f=\frac{f_{0}}{\phi}
$$

which gives

$$
p=\left(-1+\frac{2}{3 f_{0}}\right) \rho .
$$

Then we obtain dark fluid with homogeneous EoS with $w=-1+2 / 3 f_{0}$. Note that despite the fact that specific scalartensor theory and dark fluid may predict the same FRW dynamics, their physical properties may be different. For instance, Newton law, number of FRW solutions, their stability properties may significally differ in two formulations.

Another possibility to obtain the inhomogeneous EOS dark fluid is related with the modified gravity. As an illustrative example, the following action is considered:

$$
S=\int d^{4} x \sqrt{-g}\left(\frac{1}{2 \kappa^{2}} R+\mathcal{L}_{\text {matter }}+f(R)\right) .
$$

In the FRW universe, the gravitational equations are:

$$
\begin{aligned}
0= & -\frac{3}{\kappa^{2}} H^{2}+\rho-f\left(R=6 \dot{H}+12 H^{2}\right) \\
& +6\left(\dot{H}+H^{2}-H \frac{d}{d t}\right) f^{\prime}\left(R=6 \dot{H}+12 H^{2}\right) \\
0= & \frac{1}{\kappa^{2}}\left(2 \dot{H}+3 H^{2}\right)+p+f\left(R=6 \dot{H}+12 H^{2}\right) \\
& +2\left(-\dot{H}-3 H^{2}+\frac{d^{2}}{d t^{2}}+2 H \frac{d}{d t}\right) f^{\prime}\left(R=6 \dot{H}+12 H^{2}\right) .
\end{aligned}
$$

Here $\rho$ and $p$ are the energy density and the pressure coming from $\mathcal{L}_{\text {matter }}$. They may satisfy the equation of state like $p=w \rho$. One may now define the effective energy density $\tilde{\rho}$ and $\tilde{p}$ by

$$
\begin{aligned}
\tilde{\rho} \equiv & \rho-f\left(R=6 \dot{H}+12 H^{2}\right)+6\left(\dot{H}+H^{2}-H \frac{d}{d t}\right) f^{\prime}\left(R=6 \dot{H}+12 H^{2}\right), \\
\tilde{p}= & p+f\left(R=6 \dot{H}+12 H^{2}\right) \\
& +2\left(-\dot{H}-3 H^{2}+\frac{d^{2}}{d t^{2}}+2 H \frac{d}{d t}\right) f^{\prime}\left(R=6 \dot{H}+12 H^{2}\right) .
\end{aligned}
$$

Thus, it follows

$$
\begin{aligned}
\tilde{p}= & w \tilde{\rho}+(1+w) f\left(R=6 \dot{H}+12 H^{2}\right)+2\left((-1-3 w) \dot{H}-3(1+w) H^{2}+\frac{d^{2}}{d t^{2}}\right. \\
& \left.+(2+3 w) H \frac{d}{d t}\right) f^{\prime}\left(R=6 \dot{H}+12 H^{2}\right) .
\end{aligned}
$$


Especially if we consider the case that $f=f_{0} R$ with a constant $f_{0}$, we obtain

$$
\tilde{p}=w \tilde{\rho}+6 f_{0} \dot{H}+6(1+w) f_{0} H^{2} .
$$

Thus, FRW dynamics (here, the same FRW equations) of some modified gravity may be equivalent to FRW dynamics in GR coupled with the inhomogeneous EoS dark fluid. Of course, the physics of two equivalent FRW descriptions (Newton law, perturbations structure, stability of FRW solution, number of FRW solutions,etc) may be quite different 20]. In this respect, one should note that different modified gravities may also lead to the same FRW equations (see an explicit example in [48]). Nevertheless, the details of FRW dynamics will be again different as modified gravity predictions should be compared with observations (for some attempts in this direction, see [47, 49, 50]).

\section{DISCUSSION}

In summary, we presented the introduction to some classes of modified gravities considered as gravitational alternative for dark energy. Specifically, the models which include $f(R), f(G), f(R, G)$ terms or string-inspired GB-dilaton terms are considered (for recent review of higher derivative gravities, see 54 and for discussion of their experimental manifestations, see [55]). Special attention is paid to their versions where the action grows with decrease of the scalar curvature. In addition, the FRW universe filled with dark fluid with inhomogeneous EoS which may be induced by modified gravity is discussed. As it has been stressed in the introduction and as it has been explicitly demonstrated above, modified gravity is endowed with a quite rich cosmological structure: it may naturally lead to an effective cosmological constant, quintessence or phantom era in the late universe with a possible transition from deceleration to acceleration or (if necessary) the crossing of phantom divide. Moreover, some versions of modified gravity may pass the Solar System tests quite successfully.

Nevertheless, we are still missing the modified gravity theory which, from one side, is as good as General Relativity in Solar System tests and from another side contains the fully consistent explanation for current cosmic acceleration. (Note that if universe is currently entering to phantom phase, then some deviations from General Relativity are quite expectable at large distances). It is quite possible that other versions of modified gravity: string-inspired gravity with fourth-order (or higher) in $R$ corrections, theories which contain Traces of inverse Riemann tensor [51] or negative powers of squared Riemann and Ricci tensors [52] or even non-local Riemann and Ricci tensor terms (for instance, of the sort induced by Quantum Field Theory [53] with terms like $R / R_{\mu \nu} \square R^{\mu \nu}, R_{\mu \nu} R^{\mu \nu} / R \square R$, etc) or some combination of the above models with the ones under discussion should be considered as dark energy candidates as well. From another point, the next generation of precise observations should define the evolution of the cosmological parameters, and, perhaps, to rule out some of possible gravitational dark energy models leading to viable explanation of dark energy problem.

\section{Acknowledgments}

We would like to thank G. Allemandi, I. Brevik, A. Borowiec, S. Capozziello, G. Cognola, E. Elizalde, M. Francaviglia, S. Tsujikawa, M. Sasaki, M. Sami, L. Vanzo and S. Zerbini for helpful discussions on related questions. The research by SDO has been supported in part by LRSS project n4489.2006.02 (RFBR, Russia), by the project FIS2005-01181 (MEC, Spain) and by the project 2005SGR00790 (AGAUR, Catalunya, Spain).

[1] E. Copeland, M. Sami and S. Tsujikawa, arXiv:hep-th/0603057

[2] T. Padmanabhan, Phys. Repts. 380, 235 (2003) arXiv:astro-ph/0602117.

[3] V. Faraoni, E. Gunzig and P. Nardone, Fund. Cosm. Phys. 20, 121 (1999).

[4] S. Nojiri and S. D. Odintsov, Phys. Rev. D 68, 123512 (2003) arXiv:hep-th/0307288.

[5] S. Nojiri and S. D. Odintsov, Phys. Lett. B 562, 147 (2003) arXiv:hep-th/0303117.

[6] S. M. Carroll, V. Duvvuri, M. Trodden and M. S. Turner, Phys. Rev. D 70, 043528 (2004) arXiv:astro-ph/0306438; S. Capozziello, Int. J. Mod. Phys. D 11, 483 (2002); S. Capozzielo, S. Carloni and A. Troisi, arXiv:astro-ph/0303041

[7] S. Capozziello, arXiv:gr-qc/0412088 X. Meng and P. Wang, GRG 36, 1947 (2004); A. Dominguez and D. Barraco, Phys. Rev. D70, 043505 (2004); G. Allemandi, M. Francaviglia, M. Ruggiero and A. Tartaglia, arXiv:gr-qc/0506123 T. Koivisto, arXiv:gr-qc/0505128 G. Olmo, Phys. Rev. D72, 083505 (2005); I. Navarro and K. Acoleyen, arXiv:gr-qc/0506096 J. Cembranos, arXiv:gr-qc/0507039 T. Sotiriou, arXiv:gr-qc/0507027 M. Amarzguioui, O. Elgaroy, D. F. Mota and T. Multamaki, arXiv:astro-ph/0510519 C. Shao, R. Cai, B. Wang and R. Su, arXiv:gr-qc/0511034 S. Capozziello and A. Troisi, 
arXiv:astro-ph/0507545 K. Bronnikov, S. Kononogov and V. Melnikov, arXiv:gr-qc/0601114 K. Atazadeh and H. Sepangi, arXiv:gr-qc/0602028

[8] A. D. Dolgov and M. Kawasaki, Phys. Lett. B 573, 1 (2003) arXiv:astro-ph/0307285.

[9] M. E. Soussa and R. P. Woodard, GRG 36, 855 (2004); R. P. Woodard, arXiv:astro-ph/0601672

[10] S. Nojiri and S. D. Odintsov, Mod. Phys. Lett. A A19, 627 (2004) arXiv:hep-th/0310045.

[11] T. Chiba, Phys. Lett. B 575 1, (2003) arXiv:astro-ph/0307338.

[12] S. Nojiri and S. D. Odintsov, GRG 36, 1765 (2004) arXiv:hep-th/0308176.

[13] X. Meng and P. Wang, Phys. Lett. B 584, 1 (2004).

[14] M. C. B. Abdalla, S. Nojiri and S. D. Odintsov, Class. Quant. Grav. 22, L35 (2005) arXiv:hep-th/0409177.

[15] B. McInnes, JHEP 0208, 029 (2002) arXiv:hep-th/0112066.

[16] J. D. Barrow, Class. Quant. Grav. 21, L79 (2004) arXiv:gr-qc/0403084; J. D. Barrow, Class. Quant. Grav. 21, 5619 (2004) arXiv:gr-qc/0409062; S. Cotsakis and I. Klaoudatou, arXiv:gr-qc/0409022 K. Lake, Class. Quant. Grav. 21, L129 (2004) arXiv:gr-qc/0407107; M. Dabrowski, arXiv:gr-qc/0410033 L. Fernandez-Jambrina and R. Lazkoz, Phys. Rev. D 70, 121503 (2004) arXiv:gr-qc/0410124; C. Cattoen and M. Visser, arXiv:gr-qc/0508045 P. Tretyakov, A. Toporensky, Yu. Shtanov and V. Sahni, arXiv:gr-qc/0510104

[17] S. Nojiri and S. D. Odintsov, Phys. Lett. B 595, 1 (2004) arXiv:hep-th/0405078; S. Srivastava, arXiv:hep-th/0411221

[18] S. Nojiri, S. D. Odintsov and S. Tsujikawa, Phys. Rev. D 71, 063004 (2005) arXiv:hep-th/0501025.

[19] H. Štefančić, arXiv:astro-ph/0411630

[20] S. Capozziello, S. Nojiri and S. D. Odintsov, arXiv:hep-th/0512118 S. Nojiri and S. D. Odintsov, arXiv:hep-th/0506212

[21] G. Cognola, E. Elizalde, S. Nojiri, S. D. Odintsov and S. Zerbini, JCAP 0502, 010 (2005) arXiv:hep-th/0501096.

[22] V. Faraoni, arXiv:gr-qc/0511094 arXiv:gr-qc/0509008

[23] S. Das, N. Banerjee and N. Dadhich, arXiv:astro-ph/0505096

[24] I. Antoniadis, J. Rizos, K. Tamvakis, Nucl. Phys. B 415, 497 (1994) arXiv:hep-th/9305025; P. Kanti, J. Rizos and K. Tamvakis, Phys. Rev. D 59, 083512 (1999) arXiv:gr-qc/9806085.

[25] N. E. Mavromatos and J. Rizos, Phys. Rev. D 62, 124004 (2000) arXiv:hep-th/0008074; Int. J. Mod. Phys. A 18, 57 (2003) arXiv:hep-th/0205299.

[26] S. Nojiri and S. D. Odintsov, Phys. Lett. B576, 5 (2003) arXiv:hep-th/0307071; U. Gunther, A. Zhuk, V. Bezerra and C. Romero, Class. Quant. Grav. 22, 3135 (2005).

[27] S. Nojiri, S. D. Odintsov and M. Sasaki, arXiv:hep-th/0504052

[28] S. Nojiri and S. D. Odintsov, Phys. Rev. D 70, 103522 (2004) arXiv:hep-th/0408170.

[29] B. Carter and I. Neupane, arXiv:hep-th/0510109 arXiv:hep-th/0512262

[30] L. Perivolaropoulos, arXiv:astro-ph/0601014 R. Lazkoz, S. Nesseris and L. Perivolaropoulos, arXiv:astro-ph/0503230 H. Jassal, J. Bagla and T. Padmanabhan, arXiv:astro-ph/0506748 astro-ph/0601389

[31] M. Sami, A. Toporensky, P. Trejakov and S. Tsujikawa, Phys. Lett. B 619, 193 (2005) arXiv:hep-th/0504154; G. Calcagni, S. Tsujikawa and M. Sami, Class. Quant. Grav. 22, 3977 (2005) arXiv:hep-th/0505193;

[32] S. Tsujikawa, R. Brandenberger and F. Finelli, Phys. Rev. D 66, 083513 (2002).

[33] S. Nojiri and S. D. Odintsov, Phys. Lett. B 599, 137 (2004) arXiv:astro-ph/0403622 ; G. Allemandi, A. Borowiec, M. Francaviglia and S. D. Odintsov, Phys. Rev. D 72, 063505 (2005) arXiv:gr-qc/0504057.

[34] S. Capozziello, G. Lambiase and H.-J. Schmidt, Ann. Phys. (Leipzig) 9, 39 (2000).

[35] I. L. Buchbinder, S. D. Odintsov and I. L. Shapiro, Effective Action in Quantum Gravity, IOP Publishing, BristolPhiladelphia, 1992.

[36] S. Mukohyama and L. Randall, Phys. Rev. Lett. 92, 211302 (2004).

[37] A. D. Dolgov and M. Kawasaki arXiv:astro-ph/0307442

[38] T. Inagaki, S. Nojiri and S. D. Odintsov, JCAP 0506, 010 (2005) arXiv:gr-qc/0504054.

[39] S. M. Barr, S. Ng and R. Scherrer, arXiv:hep-ph/0601053

[40] T. Biswas and A. Notari, arXiv:hep-ph/0511207

[41] E. Elizalde, S. Nojiri and S. D. Odintsov, Phys. Rev. D 70, 043539 (2004) arXiv:hep-th/0405034].

[42] G. Cognola, E. Elizalde, S. Nojiri, S. D. Odintsov and S. Zerbini, arXiv:hep-th/0601008

[43] T. Clifton and J. Barrow, Phys. Rev. D 72, 103005 (2005).

[44] S. Nojiri and S. D. Odintsov, Phys. Lett. B631, 1 (2005) arXiv:hep-th/0508049; S. Nojiri, S. D. Odintsov and O. G. Gorbunova, arXiv:hep-th/0510183

[45] I. Brevik and O. Gorbunova, arXiv:gr-qc/0504001 I. Brevik, arXiv:gr-qc/0601100 M. Hu and X. Meng, arXiv:astro-ph/0511615 J. Ren and X. Meng, arXiv:astro-ph/0511163

[46] S. Nojiri and S. D. Odintsov, Phys. Rev. D 72, 023003 (2005) arXiv:hep-th/0505215.

[47] S. Capozziello,V. F. Cardone, E. Elizalde, S. Nojiri and S. D. Odintsov, arXiv:astro-ph/0508350

[48] T. Multamaki and I. Vilja, arXiv:astro-ph/0506692

[49] S. Capozziello, V. Cardone and A. Troisi, Phys. Rev. D 71, 043503 (2005); O. Mena, J. Santiago and J. Weller, arXiv:astro-ph/0510453 S. Capozziello, V. Cardone and M. Francaviglia, arXiv:astro-ph/0410135

[50] T. Koivisto and H. Kurki-Suonio, arXiv:astro-ph/0509422

[51] D. Easson, F. Schuller, M. Trodden and M. Wohlfarth, arXiv:astro-ph/0506392 F. Schuller and M. Wohlfarth, gr-qc/0411076

[52] S. Carroll, A. De Felice, V. Duvvuri, D. Easson, M. Trodden and M. Turner, Phys. Rev. D 71, 063513 (2005); G. Allemandi, A. Borowiec and M. Francaviglia, Phys. Rev. D 70, 103503 (2004).

[53] E. Elizalde and S. D. Odintsov, Mod. Phys. Lett. A A10, 1821 (1995), arXiv:gr-qc/9508041. 
[54] H.-J. Schmidt, arXiv:gr-qc/0602017

[55] T. Rizzo, arXiv:hep-ph/0412251 arXiv:hep-ph/0510420 